Article

\title{
Fuzzy Static Output Control of T-S Fuzzy Stochastic Systems via Line Integral Lyapunov Function
}

\author{
Cheung-Chieh $\mathrm{Ku} * \mathbb{D}$, Yun-Chen Yeh, Yann-Hong Lin and Yu-Yen Hsieh \\ Department of Marine Engineering, National Taiwan Ocean University, Keelung 202, Taiwan; \\ e572130102@gmail.com (Y.-C.Y.); ginobili9815318@gmail.com (Y.-H.L.); infinite7321@gmail.com (Y.-Y.H.) \\ * Correspondence: ccku@mail.ntou.edu.tw
}

Citation: Ku, C.-C.; Yeh, Y.-C.; Lin, Y.-H.; Hsieh, Y.-Y. Fuzzy Static Output Control of T-S Fuzzy Stochastic Systems via Line Integral Lyapunov Function. Processes 2021, 9, 697. https://doi.org/10.3390/pr9040697

Academic Editor: Jie Zhang

Received: 7 March 2021

Accepted: 7 April 2021

Published: 15 April 2021

Publisher's Note: MDPI stays neutral with regard to jurisdictional claims in published maps and institutional affiliations.

Copyright: (c) 2021 by the authors. Licensee MDPI, Basel, Switzerland. This article is an open access article distributed under the terms and conditions of the Creative Commons Attribution (CC BY) license (https:// creativecommons.org/licenses/by/ $4.0 /)$.

\begin{abstract}
Considering some unmeasurable states, a fuzzy static output control problem of nonlinear stochastic systems is discussed in this paper. Based on a modelling approach, a Takagi-Sugeno (T-S) fuzzy system, constructed by a family of stochastic differential equations and membership functions, is applied to represent nonlinear stochastic systems. Parallel distributed compensation (PDC) technology is used to construct the static output controller. A line-integral Lyapunov function (LILF) is used to derive some sufficient conditions for guaranteeing the asymptotical stability in the mean square. From the LILF, a potential conservatism produced by the derivative of the membership function is eliminated to increase the relaxation of sufficient conditions. Furthermore, those conditions are transferred into linear matrix inequality (LMI) form via projection lemma. According to the convex optimization algorithm, the feasible solutions are directly obtained to establish the static output fuzzy controller. Finally, a numerical example is applied to demonstrate the effectiveness and usefulness of the proposed design method.
\end{abstract}

Keywords: T-S fuzzy systems; static output control; stochastic system; line-integral Lyapunov function; projection lemma

\section{Introduction}

The fuzzy set provides a concept differing from the crisp set for discussing the problems of economics, management, and control. During fuzzification, the states in the nonlinearity are usually premise variables. Moreover, the linear systems and the membership function can be determined via the chosen equilibrium points to build the T-S fuzzy system. Furthermore, the center of gravity method [1] is a common defuzzification technique method and is used to convert the fuzzified output into a single crisp value with respect to a fuzzy set. Referring to [2], the fuzzy set was applied to formulate the mathematical model of optimization of sustainable waste management. In the past decades, the Takagi-Sugeno (T-S) fuzzy system has been widely used by blending some subsystems and the specific membership function to represent nonlinear systems. In the literature [3-13], several results for the control problem of nonlinear systems have been already dealt with using linear control theories through the T-S fuzzy system. T-S fuzzy systems have also been investigated for their use in stability issues of practical systems such as turbocharged gasoline engines [9], flexible wings [10], and vessel dynamic positioning [11]. To develop the controller design method for T-S fuzzy systems, the parallel distributed compensation (PDC) concept was first proposed [12]. Similar to the structure of the T-S fuzzy system, the PDC technique involves setting up a unique subcontroller for each linear subsystem and blending them with a specific membership function. Referring to the literature [5,6,9-12], the PDC concept has been applied successfully and widely to design corresponding fuzzy controllers. Most of the existing PDC-based controller design methods [5,6,9-12] were proposed using a state-feedback scheme. The control problems of nonlinear systems with unmeasurable states were discussed by designing observer-based controllers [13,14], a dynamic output controller [15], and a static output controller [16]. It is well-known that the 
static output feedback control scheme [16] is a reasonable and easy method for solving the stabilization issue of systems with unmeasured states. Because of this, the control problem of the output feedback technique, which uses measurable states as feedback signals, becomes a worthy issue for discussion and investigation regarding T-S fuzzy systems.

The output control problems of polynomial systems, such as the T-S fuzzy system $[17,18]$ and the linear parameter varying system [19], usually become complex and difficult according to the number of sufficient conditions. Some potential conservatisms for the static output feedback controller design method are caused by separating the variables to convert the sufficient conditions into linear matrix inequalities (LMIs). To propose the static output controller design method, some equalities are required [18] such that the derived conditions belong to LMI problems. However, those equalities cause some restrictions for specifying the positive definite matrix when designing the static output controller. An iterative LMI algorithm $[20,21]$ has been proposed to deal with the static output control problem described by the LMI form. Thus, a parameter-dependent Lyapunov function has been applied [22,23] to develop some relaxed stability conditions. The parameterdependent Lyapunov function is composed of several positive definite matrices that can relax the restrictions in finding a common positive definite matrix. Even though relaxed conditions can be obtained by a parameter-dependent Lyapunov function, the derivative of the membership function is necessarily bound, causing a potential conservatism $[22,23]$ in the stability analysis. A line-integral Lyapunov function (LILF) was proposed to avoid the conservatism caused by the derivative of the membership function, and to keep the relaxation caused by the multiple positive definite matrices [24]. Relaxed fuzzy controller design methods have been developed via the LILF [24-26]. Although the LILF provides relaxations in the stability criterion, the special structure of the positive definite matrix is dependent on the premise variables. The structure of the positive definite matrix creates difficulties when deriving the LMI form of sufficient conditions. In the literature [27], an iterative LMI algorithm was proposed to search for feasible solutions. Generally, the iterative LMI algorithm provides conservatism caused by linear transformations or inequalities. Extending the results of [22], the stability criterion was therefore developed via guaranteeing the stability and performance of the T-S fuzzy stochastic systems based on LILF in this paper.

For stochastic systems, several works [28-32] have discussed their stability and stabilization problems. Some results for stability criteria of nonlinear stochastic systems were developed by the T-S fuzzy system and the Itô equation. The Itô equation is regarded as the combination of a common differential equation and a multiplicative noise term [28]. The stability of stochastic systems is based on the concept of root mean square because of their unpredictability [28-32]. The Itô formula [28-32] is usually applied to derive some sufficient conditions. Based on the Itô formula, some static output feedback controller design methods have been proposed by [28-30] so that the stability of nonlinear stochastic systems is guaranteed in the mean square. In the literature [31], the parameter-independent Lyapunov function was selected to develop their stability criterion. The relaxed output feedback controller design methods for polynomial stochastic systems [32] were proposed via applying the parameter-dependent Lyapunov function. Generally, the convex optimization algorithm [33] is an efficient method for solving the control problems; moreover, it is widely employed in discussions of the stabilization problem of T-S fuzzy systems. However, the algorithm requires the sufficient condition being converted into LMI form [33]. Because of the output feedback scheme, the stabilization problem belongs to the strict bilinear form that is hard to convert into LMI form. Thus, some equalities [27] and extra algorithms $[20,21]$ have been proposed such that the bilinear problem can be calculated via the convex optimization algorithm. However, potential conservatism was caused during use of the technologies and extra algorithm. Thus, a relaxed stability criterion is an important issue so that the nonlinear stochastic system is asymptotically stable in the mean square. 
Based on the above motivation, an output feedback control problem of the nonlinear stochastic system is discussed in this paper. With reference to the modeling approach and the Itô differential equation, the T-S fuzzy system with multiplicative noise terms can be constructed to represent the nonlinear stochastic system. Using the PDC concept, the static output feedback controller is established to deal with the stabilization problem of the considered system with unmeasurable states. For the stabilization problem, the LILF is chosen to derive some sufficient conditions such that the potential conservatism produced by the derivative of the membership function is eliminated. Some technologies [14,34] are applied to convert the sufficient conditions into a strict LMI form that can be directly solved by the convex optimization algorithm. Through solving the conditions, the feedback gains can be obtained to design a static output controller to guarantee the asymptotical stability in the mean square. Finally, a numerical is provided to verify the effectiveness and application of the proposed static output controller design method.

This paper is structured as follows: In Section 2, the output feedback stability problem of nonlinear stochastic system is investigated via the T-S fuzzy system. In Section 3, some sufficient conditions are derived by the LILF and Itô formula. In Section 4, the simulation results of a numerical example are presented. In Section 5, some conclusions are given.

I represents the identity matrix with appropriate dimension, $E\{\cdot\}$ represents the expected value of $\cdot \operatorname{He}\{\cdot\}$ represents the $[\cdot]+[\cdot]^{T}$, and $*$ represents the symmetric parts in the block matrix.

\section{System Descriptions and Problem Statements}

According to the T-S fuzzy system and Itô equation, the nonlinear stochastic system can be described as follows:

If $x_{1}(t)$ is $M_{1}^{\alpha i 1}$ and $x_{2}(t)$ is $M_{2}^{\alpha i 2}$ and $\ldots$ and $x_{n}(t)$ is $M_{n}^{\alpha i n}$, then

$$
\begin{gathered}
d x(t)=\left(\mathbf{A}_{i} x(t)+\mathbf{B}_{i} u(t)\right) d t+\left(\overline{\mathbf{A}}_{i} x(t)+\overline{\mathbf{B}}_{i} u(t)\right) d \beta(t) \\
y(t)=\mathbf{C}_{i} x(t)
\end{gathered}
$$

where $x^{\mathrm{T}}(t) \in \mathbb{R}^{n}$ is the state vector, $u^{\mathrm{T}}(t) \in \mathbb{R}^{m}$ is the control input vector, $y(t) \in \mathbb{R}^{q}$ is the measured output, and $M_{n}^{\alpha i j}$ are the fuzzy sets for $i=1,2, \ldots, N$. Then $\alpha_{i j}$ is the scalar representing the $x_{j}$-based fuzzy set used in the $i$ th fuzzy rule. $\beta(t)$ is a scalar continuous-type Brownian motion [30] satisfying the independent increment properties as $E\{d \beta(t)\}=E\{x(t) d \beta(t)\}=0$ and $E\{d \beta(t) d \beta(t)\}=1 . \mathbf{A}_{i}, \mathbf{B}_{i}, \overline{\mathbf{A}}_{i}, \overline{\mathbf{B}}_{i}$, and $\mathbf{C}_{i}$ are constant matrices with compatible dimension. Thus, the system in (1)-(2) can be furtherly represented as follows:

$$
\begin{gathered}
d x(t)=\sum_{i=1}^{N} h_{i}(t)\left\{\left(\mathbf{A}_{i} x(t)+\mathbf{B}_{i} u(t)\right) d t+\left(\overline{\mathbf{A}}_{i} x(t)+\overline{\mathbf{B}}_{i} u(t)\right) d \beta(t)\right\} \\
y(t)=\sum_{i=1}^{N} h_{i}(t) \mathbf{C}_{i} x(t)
\end{gathered}
$$

where $\mu_{j}^{\alpha_{i j}}\left(x_{j}(t)\right)=\frac{\mu_{j}^{\alpha_{i j}}(x(t))}{\sum_{i=1}^{N} \mu_{j}^{\alpha_{i j}}(x(t))}, h_{i}(x(t))=\prod_{j=1}^{n} \mu_{j}^{\alpha i j}\left(x_{j}(t)\right), 0 \leq h_{i}(x(t)) \leq 1$ and $\sum_{i=1}^{N} h_{i}(x(t))=1$.

Based on the PDC concept, the following output controller is considered for the system in (3)-(4):

If $x_{1}(t)$ is $M_{1}^{\alpha i 1}$ and $x_{2}(t)$ is $M_{2}^{\alpha i 2}$ and $\ldots$ and $x_{n}(t)$ is $M_{n}^{\alpha i n}$ then

$$
u(t)=-\mathbf{F}_{i} y(t)
$$


or

$$
u(t)=\sum_{i=1}^{N} h_{i}(t)\left(-\mathbf{F}_{i} y(t)\right)
$$

The following closed-loop system can be constructed by substituting the output controller in (6) into (3)-(4).

$$
\begin{gathered}
d x(t)=\sum_{i=1}^{N} \sum_{j=1}^{N} \sum_{k=1}^{N} h_{i}(t) h_{j}(t) h_{k}(t)\left\{\left(\mathbf{A}_{i}-\mathbf{B}_{i} \mathbf{F}_{j} \mathbf{C}_{k}\right) x(t) d t+\left(\overline{\mathbf{A}}_{i}-\overline{\mathbf{B}}_{i} \mathbf{F}_{\mathbf{j}} \mathbf{C}_{k}\right) x(t) d \beta(t)\right\} \\
=\sum_{i=1}^{N} \sum_{j=1}^{N} \sum_{k=1}^{N} h_{i}(t) h_{j}(t) h_{k}(t)\left\{\mathbf{R}_{i j k} x(t) d t+\overline{\mathbf{R}}_{i j k} x(t) d \beta(t)\right\}
\end{gathered}
$$

where $\mathbf{R}_{i j k}=\mathbf{A}_{i}-\mathbf{B}_{i} \mathbf{F}_{j} \mathbf{C}_{k}$ and $\overline{\mathbf{R}}_{i j k}=\overline{\mathbf{A}}_{i}-\overline{\mathbf{B}}_{i} \mathbf{F}_{j} \mathbf{C}_{k}$.

Due to the stochastic behaviors, the following definition is applied to ensure the stability of the closed-loop system in (7):

Definition 1 [30]. For the closed-loop system in (7), the solution is asymptotically stable in the mean square when $E\{x(t)\}$ and $E\left\{x^{T}(t) x(t)\right\}$ are converged to zero as $t \rightarrow \infty$.

To solve the stabilization problem, several useful lemmas are applied as follows so that the derived conditions can be converted into LMI form.

Lemma 1 [34]. For some given matrices $\mathbf{K}=\mathbf{K}^{\mathbf{T}}>0, \mathbf{Z}=\mathbf{Z}^{\mathbf{T}}$ and $\mathbf{Y}$, there exists a matrix $\mathbf{H}$ such that the following conditions are equivalent:

$$
\mathbf{K}+\mathbf{Y}^{\mathbf{T}} \mathbf{Z Y}<0
$$

and

$$
\left[\begin{array}{cc}
\mathbf{K} & * \\
\mathbf{H Y} & -\mathbf{H}-\mathbf{H}^{\mathrm{T}}+\mathbf{Z}
\end{array}\right]<0
$$

Lemma 2 [14]. Giving the matrices, $\boldsymbol{\Phi}=\boldsymbol{\Phi}^{\mathrm{T}} \in \Re^{n_{\boldsymbol{\Phi}} \times n_{\boldsymbol{\Phi}}}, \boldsymbol{\psi} \in \Re^{n_{\boldsymbol{\Psi}} \times n_{\boldsymbol{\Phi}}}$, and $\boldsymbol{\Lambda} \in \Re^{n_{\boldsymbol{\Lambda}} \times n_{\boldsymbol{\Phi}}}$, which satisfy $\operatorname{rank}(\boldsymbol{\Psi})<n_{\boldsymbol{\Phi}}$ and $\operatorname{rank}(\boldsymbol{\Lambda})<n_{\boldsymbol{\Phi}}$, if there exists the matrix $X$ such that

$$
\left[\begin{array}{cc}
\boldsymbol{\Xi}+\mathbf{G}-H e\{\mathbf{J}\} & * \\
\boldsymbol{\psi}^{\mathrm{T}} \mathbf{X} \mathbf{\Lambda}+\mathbf{J} & -\mathbf{G}
\end{array}\right]<0
$$

then the following inequalities are held:

$$
\boldsymbol{\psi}_{\perp}^{\mathrm{T}} \boldsymbol{\Phi} \psi_{\perp}<0 \text { and } \boldsymbol{\Lambda}_{\perp}^{\mathrm{T}} \boldsymbol{\Phi} \boldsymbol{\Lambda}_{\perp}<0
$$

where $\boldsymbol{\psi}_{\perp}^{\mathrm{T}}$ and $\boldsymbol{\Lambda}_{\perp}^{\mathrm{T}}$ are the matrices whose columns form a basis of the null-space of $\boldsymbol{\psi}$ and $\boldsymbol{\Lambda}$, respectively.

In the next section, a stability criterion is proposed to ensure the closed-loop system in (7) is asymptotically stable in the mean square. Some sufficient conditions described in an LMI form are derived to find the gains for designing the fuzzy controller in (6).

\section{Stability Criterion with Output Feedback Controller}

In this section, the LILF and Itô formula are employed to derive some sufficient conditions and to avoid the potential conservatism caused by the derivative of the membership function. The lemmas are used to convert the conditions into an LMI form so that the feasible solutions can be directly obtained by the convex optimization algorithm. With the solutions, the PDC-based output controller in (6) can be established to guarantee the asymptotical stability of the closed-loop system, shown in (7), in the mean square. 
Theorem 1. The closed-loop system (7) is asymptotically stable in the mean square if there exist feedback gains $\mathbf{F}_{i}$ and positive definite matrices $\hat{\mathbf{P}}_{i}$ such that

$$
\begin{gathered}
\mathbf{R}_{i j k}^{\mathrm{T}} \hat{\mathbf{P}}_{i}+\hat{\mathbf{P}}_{i} \mathbf{R}_{i j k}+\overline{\mathbf{R}}_{i j k}^{\mathrm{T}} \hat{\mathbf{P}}_{i} \overline{\mathbf{R}}_{i j k}<0, i, j, k=1,2, \cdots, N \\
\text { where } \hat{\mathbf{P}}_{i}=\mathbf{D}_{i}+\overline{\mathbf{P}}, \overline{\mathbf{P}}=\left[\begin{array}{cccc}
0 & p_{12} & \cdots & p_{1 n} \\
p_{12} & 0 & \cdots & p_{2 n} \\
\vdots & \vdots & \ddots & \vdots \\
p_{1 n} & p_{2 n} & \cdots & 0
\end{array}\right] \text { and } \mathbf{D}_{i}=\left[\begin{array}{cccc}
d_{11}^{\alpha i 1} & 0 & \cdots & 0 \\
0 & d_{22}^{\alpha i 2} & \cdots & 0 \\
\vdots & \vdots & \ddots & \vdots \\
0 & 0 & \cdots & d_{n n}^{\alpha i n}
\end{array}\right]
\end{gathered}
$$

Proof. To avoid the conservatism caused by the derivative of the membership function, the following LILF [24] is used to develop the stability criterion for the closed-loop system in (7).

$$
V(x(t))=2 \int_{\Gamma(0, x)} f(\varphi) \cdot d \varphi
$$

where $\Gamma(0, x)$ is a path from the origin to the current state, $\varphi$ is a dummy vector for the integral, and $f(\varphi)=\hat{\mathbf{P}}_{i}(x(t)) x(t)=\sum_{i=1}^{N} h_{i}(t) \hat{\mathbf{P}}_{i} x(t)$.

Applying Itô's formula, we find the following derivation of $V(x(t))$ in (12) along the trajectories of (7)

$$
d V(x(t))=L V(x(t)) d t+2 \sum_{i=1}^{N} \sum_{j=1}^{N} \sum_{k=1}^{N} h_{i}(t) h_{j}(t) h_{k}(t)\left\{x^{T}(t) \hat{\mathbf{P}}_{i} \overline{\mathbf{R}}_{i j k} x(t) d \beta(t)\right\}
$$

where

$$
L V(x(t))=\sum_{i=1}^{N} \sum_{j=1}^{N} \sum_{k=1}^{N} h_{i}(t) h_{j}(t) h_{k}(t)\left\{x^{T}(t)\left(\mathbf{R}_{i j k}^{T} \hat{\mathbf{P}}_{i}+\hat{\mathbf{P}}_{i} \mathbf{R}_{i j k}+\overline{\mathbf{R}}_{i j k}^{T} \hat{\mathbf{P}}_{i} \overline{\mathbf{R}}_{i j k}\right) x(t)\right\}
$$

Taking the expectation of (13), the following equation can be inferred due to the independent increment property of $\beta(t)$.

$$
E\{d V(x(t))\}=E\{L V(x(t)) d t\}
$$

Obviously, if (11) holds, then $E\{L V(x(t))\}<0$ can be obtained from (14). Since $E\{L V(x(t))\}<0, E\{d V(x(t))\}<0$ can be further found by (15). Referring to the Definition and $E\{d V(x(t))\}<0$, the asymptotical stability of the closed-loop system in (7) is guaranteed in the mean square.

To establish the controller in (6), the feasible solutions as $\mathbf{F}_{i}$ and $\hat{\mathbf{P}}_{i}$ are required to satisfy Theorem 1 . Nevertheless, the condition shown in (11) in Theorem 1 cannot be directly solved by the convex optimization algorithm due to the bilinear terms composed by $\mathbf{F}_{i}$ and $\hat{\mathbf{P}}_{i}$. Therefore, the lemmas are applied to find the corresponding LMI form of (11).

Theorem 2. The closed-loop system in (7) is asymptotically stable in the mean square if there exist matrices $\mathbf{X}, \mathbf{F}_{j}, \mathbf{H}$, and $\mathbf{J}_{i}$, and positive definite matrices $\hat{\mathbf{P}}_{i}$ and $\mathbf{G}_{i}$ such that

$$
\left[\begin{array}{cc}
\Xi_{i j k}+\mathrm{G}_{i}-H e\left\{\mathbf{J}_{i}\right\} & * \\
\mathrm{~T}_{i j k}+\mathrm{J}_{i} & -\mathrm{G}_{i}
\end{array}\right]<0, i, j, k=1,2, \ldots, N
$$




$$
\text { where } \mathbf{\Xi}_{i j k}=\left[\begin{array}{cccc}
0 & \hat{\mathbf{P}}_{i} & 0 & 0 \\
\hat{\mathbf{P}}_{i} & -2 \hat{\mathbf{P}}_{i} & \overline{\mathbf{R}}_{i j k}^{\mathrm{T}} & 0 \\
0 & \overline{\mathbf{R}}_{i j k} & 0 & -\mathbf{I} \\
0 & 0 & -\mathbf{I} & \hat{\mathbf{P}}_{i}
\end{array}\right] \text { and } \mathbf{T}_{i j k}=\left[\begin{array}{cccc}
-\mathbf{I} & 0 & 0 & 0 \\
\mathbf{R}_{i j k}^{\mathrm{T}}+\mathbf{I} & 0 & 0 & 0 \\
0 & 0 & -\mathbf{X} & \mathbf{X} \\
0 & 0 & \mathbf{Q} & -\mathbf{Q}
\end{array}\right] \text { with } \mathbf{Q}=
$$
HX.

Proof. Firstly, the following inequality can be inferred from (16).

$$
\left[\begin{array}{cc}
\mathbf{\Xi}_{i j k}+\mathbf{G}_{i}-H e\left\{\mathbf{J}_{i}\right\} & * \\
\boldsymbol{\psi}_{i j k}^{\mathrm{T}} \hat{\mathbf{X}} \boldsymbol{\Lambda}_{i j k}+\mathbf{J}_{i} & -\mathbf{G}_{i}
\end{array}\right]<0
$$

where $\boldsymbol{\psi}_{i j k}=\left[\begin{array}{cccc}-\mathbf{I} & \mathbf{R}_{i j k}+\mathbf{I} & 0 & 0 \\ 0 & 0 & -\mathbf{I} & \mathbf{H}^{\mathrm{T}}\end{array}\right], \boldsymbol{\Lambda}_{i j k}=\left[\begin{array}{cccc}\mathbf{I} & 0 & 0 & 0 \\ 0 & 0 & \mathbf{I} & -\mathbf{I}\end{array}\right]$, and $\hat{\mathbf{X}}=\left[\begin{array}{cc}\mathbf{I} & 0 \\ 0 & \mathbf{X}\end{array}\right]$.

Applying Lemma 2 and (17), we find the following inequalities by choosing the null-space matrices $\boldsymbol{\psi}_{i j k \perp}^{\mathrm{T}}$ and $\boldsymbol{\Lambda}_{i j k \perp}^{\mathrm{T}}$.

$$
\boldsymbol{\psi}_{i j k \perp}^{\mathrm{T}} \boldsymbol{\Xi}_{i j k} \boldsymbol{\psi}_{i j k \perp}<0
$$

and

$$
\boldsymbol{\Lambda}_{i j k \perp}^{\mathrm{T}} \boldsymbol{\Xi}_{i j k} \boldsymbol{\Lambda}_{i j k \perp}<0
$$

where

$$
\boldsymbol{\psi}_{i j k \perp}^{\mathrm{T}}=\left[\begin{array}{cccc}
\mathbf{R}_{i j k}^{\mathrm{T}}+\mathbf{I} & \mathbf{I} & 0 & 0 \\
0 & 0 & \mathbf{H} & \mathbf{I}
\end{array}\right], \boldsymbol{\Lambda}_{i j k \perp}^{\mathrm{T}}=\left[\begin{array}{cccc}
0 & \mathbf{I} & 0 & 0 \\
0 & 0 & \mathbf{I} & \mathbf{I}
\end{array}\right] \text { and } \boldsymbol{\Xi}_{i j k}=\left[\begin{array}{cccc}
0 & \hat{\mathbf{P}}_{i} & 0 & 0 \\
\hat{\mathbf{P}_{i}} & -2 \hat{\mathbf{P}}_{i} & \overline{\mathbf{R}}_{i j k}^{\mathrm{T}} & 0 \\
0 & \overline{\mathbf{R}}_{i j k} & 0 & -\mathbf{I} \\
0 & 0 & -\mathbf{I} & \hat{\mathbf{P}}_{i}
\end{array}\right]
$$

Based on Lemma 1, if there exists a matrix $\mathbf{H}$ satisfying the following condition, then the condition in (11) in Theorem 1 holds.

$$
\left[\begin{array}{cc}
\mathbf{R}_{i j k}^{\mathrm{T}} \hat{\mathbf{P}}_{i}+\hat{\mathbf{P}}_{i} \mathbf{R}_{i j k} & \overline{\mathbf{R}}_{i j k}^{\mathrm{T}} \mathbf{H}^{\mathrm{T}} \\
\mathbf{H} \overline{\mathbf{R}}_{i j k} & -\mathbf{H}-\mathbf{H}^{\mathrm{T}}+\hat{\mathbf{P}}_{i}
\end{array}\right]<0
$$

Arranging (18), we can directly find the following inequality:

$$
\boldsymbol{\psi}_{i j k \perp}^{\mathrm{T}} \boldsymbol{\Xi}_{i j k} \boldsymbol{\psi}_{i j k \perp}=\left[\begin{array}{cc}
\mathbf{R}_{i j k}^{\mathrm{T}} \hat{\mathbf{P}}_{i}+\hat{\mathbf{P}}_{i} \mathbf{R}_{i j k} & \overline{\mathbf{R}}_{i j k}^{\mathrm{T}} \mathbf{H}^{\mathrm{T}} \\
\mathbf{H} \overline{\mathbf{R}}_{i j k} & -\mathbf{H}-\mathbf{H}^{\mathrm{T}}+\hat{\mathbf{P}}_{i}
\end{array}\right]<0
$$

Form (21), the inequality in (20) is equal to (18). Thus, if the condition in (16) holds, then the condition in (11) in Theorem 1 is also satisfied. According to Theorem 1, the closed-loop T-S fuzzy system in (7) is asymptotically stable in the mean square. In addition, the inequality in (19) also holds because (17) and Lemma 1 can be further demonstrated by the simulated results. The proof of Theorem 2 is completed.

According to the lemmas, the conditions in Theorem 1 are transferred into LMI form shown by the conditions in Theorem 2. Therefore, the feasible solutions for Theorem 2 can be directly found via the convex optimization algorithm. With the obtained solutions, the static output controller in (6) is established to ensure the asymptotical stability of the closed-loop system, as shown in (7), in the mean square. To demonstrate the proposed method, a numerical simulation and some comparisons are proposed in the following section. 


\section{Numerical Simulation}

In this section, two cases are proposed to show the contribution of this paper. In Case 1 , the method of [18] is applied to discuss the conservatism of the proposed design method. In Case 2, a comparison between the method of [18] and the proposed method is provided to show the importance of considering stochastic behavior. Consider the following T-S fuzzy system:

$$
\begin{aligned}
& d x(t)=\sum_{i=1}^{2} h_{i}(t)\left\{\left(\mathbf{A}_{i} x(t)+\mathbf{B}_{i} u(t)\right) d t+\left(\overline{\mathbf{A}}_{i} x(t)+\overline{\mathbf{B}}_{i} u(t)\right) d \beta(t)\right\} \\
& y(t)=\sum_{i=1}^{2} h_{i}(t) \mathbf{C}_{i} x(t) \\
& \text { where } \mathbf{A}_{1}=\left[\begin{array}{cccc}
0.56+\Delta & 0 & 0.4 & -0.2 \\
0 & -0.4 & -0.1 & -0.5 \\
0 & 0.2 & -0.1 & 0 \\
0.1 & 0 & 0.36+\Delta & -0.4
\end{array}\right], \mathbf{B}_{1}=\mathbf{B}_{2}=\left[\begin{array}{c}
1.5 \\
0 \\
0.1 \\
0.2
\end{array}\right], \mathbf{A}_{2}= \\
& {\left[\begin{array}{cccc}
0.56+\Delta & 0 & 0.4 & -0.2 \\
0 & -0.4 & -0.1 & -0.5 \\
0 & 0.2 & -0.1 & 0 \\
0.1 & 0 & 0.36+\Delta & -0.4
\end{array}\right], \mathbf{C}_{1}=\mathbf{C}_{2}=\left[\begin{array}{llll}
1 & 0 & 0 & 0
\end{array}\right], \overline{\mathbf{A}}_{1}=\overline{\mathbf{A}}_{2}=} \\
& {\left[\begin{array}{cccc}
0.6 & 0 & 0 & 0 \\
0 & 0.5 & 0 & 0 \\
0 & 0 & -0.1 & 0 \\
0 & 0 & 0 & 0.1
\end{array}\right] \text {, and } \overline{\mathbf{B}}_{1}=\overline{\mathbf{B}}_{2}=\left[\begin{array}{c}
0.2 \\
0 \\
0 \\
0
\end{array}\right]}
\end{aligned}
$$

where $\Delta$ is a given scalar. By setting $\Delta$, the conservatism of the proposed design method and the method of [18] can be discussed by searching the feasible solutions. In this simulation, $x_{2}(t) \in\left[0, \pm \frac{\pi}{6}\right]$ is assumed as the premise variable for building the following membership function and $x_{1}(t)$ is used as the feedback signal of the designed static output controller.

$$
h_{1}\left(x_{2}(t)\right)=\left\{1-\frac{1}{1+e^{-7\left(x_{2}(t)-\pi / 4\right)}}\right\} \times \frac{1}{1+e^{-7\left(x_{2}(t)+\pi / 4\right)}} \text { and } h_{2}\left(x_{2}(t)\right)=1-h_{1}\left(x_{2}(t)\right) .
$$

Case 1. Through ignoring the multiplicative noise term in the system (22)-(23), the method of [18] and Theorem 2 are separately applied to find the feasible solutions with the maximum value of $\Delta$. By using the method of [18], the maximum value of $\Delta$ is 0.004 . The maximum value of $\Delta$ found by Theorem 2 is 0.009 . It is obvious that the bigger value of $\Delta$ is found by the proposed design method than the one found by [18]. Thus, this paper provides a less conservative design method than [18]. Moreover, if the number of premise variables is increased, then the relaxation of the proposed design method is manifested.

Case 2. With $\Delta=-0.16$, the method of [18] is applied to design the following PDC-based output controller for the system (22)-(23).

$$
u(t)=\sum_{i=1}^{2} h_{i}\left(x_{2}(t)\right)\left(-\mathbf{F}_{i} y(t)\right)
$$

where $\mathbf{F}_{1}=0.5710$ and $\mathbf{F}_{2}=0.5710$. Based on the controller in (24), the response of $x_{1}(t)$ of the system (22)-(23) is stated in Figure 1 with the initial condition as $x(0)=$ $\left[\begin{array}{llll}0.2 & -0.75 & 0.3 & 0.55\end{array}\right]$. The following feasible solutions are found by satisfying the condition in (16) in Theorem 2: 


$$
\begin{aligned}
\mathbf{P}_{1} & =\left[\begin{array}{cccc}
1.1004 & 0.0392 & -0.0317 & 0.0035 \\
0.0392 & 0.9380 & 0.0157 & 0.0177 \\
-0.0317 & 0.0157 & 0.9334 & -0.0051 \\
0.0035 & 0.0177 & -0.0051 & 0.9375
\end{array}\right], \mathbf{F}_{1}=\left[\begin{array}{ll}
-0.1216 & 1.7736
\end{array}\right] \\
\mathbf{P}_{2} & =\left[\begin{array}{cccc}
1.1004 & 0.0392 & -0.0317 & 0.0035 \\
0.0392 & 0.9569 & 0.0157 & 0.0177 \\
-0.0317 & 0.0157 & 0.9334 & -0.0051 \\
0.0035 & 0.0177 & -0.0051 & 0.9375
\end{array}\right] \text { and } \mathbf{F}_{2}=\left[\begin{array}{lll}
-0.1205 & 1.8149
\end{array}\right] .
\end{aligned}
$$

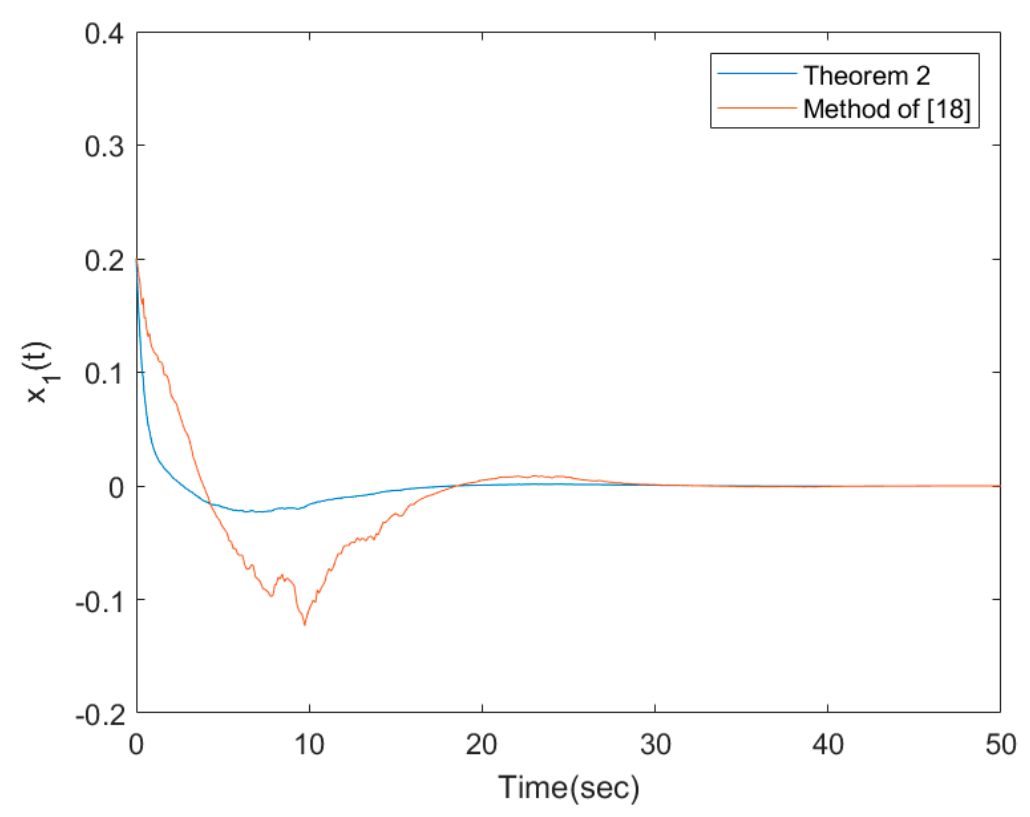

Figure 1. Comparison between this paper's method and [18].

To demonstrate the satisfaction of (19), the obtained feasible solutions in (25) are applied to ensure the following inequality:

$$
\boldsymbol{\Lambda}_{i j k \perp}^{\mathrm{T}} \boldsymbol{\Xi}_{i j k} \boldsymbol{\Lambda}_{i j k \perp}=\left[\begin{array}{cc}
-2 \mathbf{P}_{i} & \overline{\mathbf{R}}_{i j k}^{\mathrm{T}} \\
\overline{\mathbf{R}}_{i j k} & \mathbf{P}_{i}-2 \mathbf{I}
\end{array}\right]<0 .
$$

Based on the solutions in (25), the PDC-based output feedback controller is designed as follows:

$$
u(t)=\sum_{i=1}^{2} h_{i}\left(x_{2}(t)\right)\left(-\mathbf{F}_{i} y(t)\right)
$$

Along with the designed controller in (26), the responses of $x_{1}(t)$ of the system (22)-(23) are also presented in Figure 1 with the same condition. Referring to Figure 1, although stability can be achieved, the poor response of the system (22)-(23) driven by the controller in (26) is provided according to the stochastic behavior. Moreover, the transient response of the system (22)-(23) driven by the designed controller in (26) is better than one driven by (24).

Regarding robustness, the responses of the system (22)-(23) driven by the fuzzy controller in (26) are stated with three initial conditions as $x(0)=\left[\begin{array}{llll}0.2 & -0.75 & 0.3 & 0.55\end{array}\right]$, $x(0)=\left[\begin{array}{llll}0.55 & -0.9 & 0.45 & 0.75\end{array}\right]$, and $x(0)=\left[\begin{array}{llll}0.8 & -1 & 0.6 & 0.9\end{array}\right]$ in Figures 2-6. As shown in Figures 2-6, all states of (22)-(23) with different initials converge to zero, although the only measured output signals are used as feedback signals. The vibrations in those figures are caused by their stochastic behavior. Thus, the proposed design method is less conservative than the method of [18]. Moreover, the system (22)-(23) driven by the controller in (26) provides better transient response than the one driven by the controller designed by the method of [18]. 


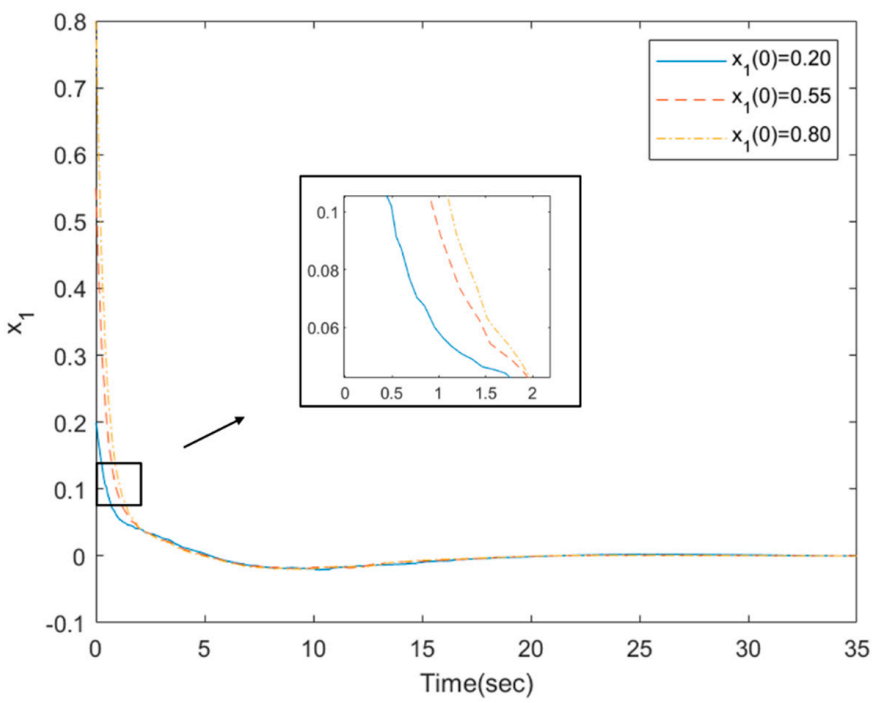

Figure 2. Response of $x_{1}(t)$.

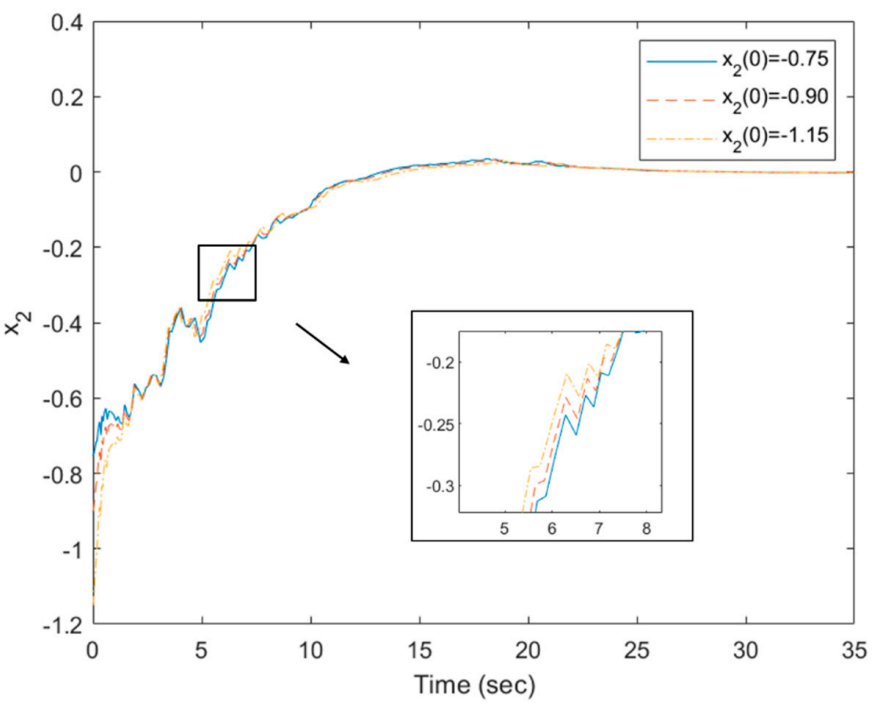

Figure 3. Response of $x_{2}(t)$.

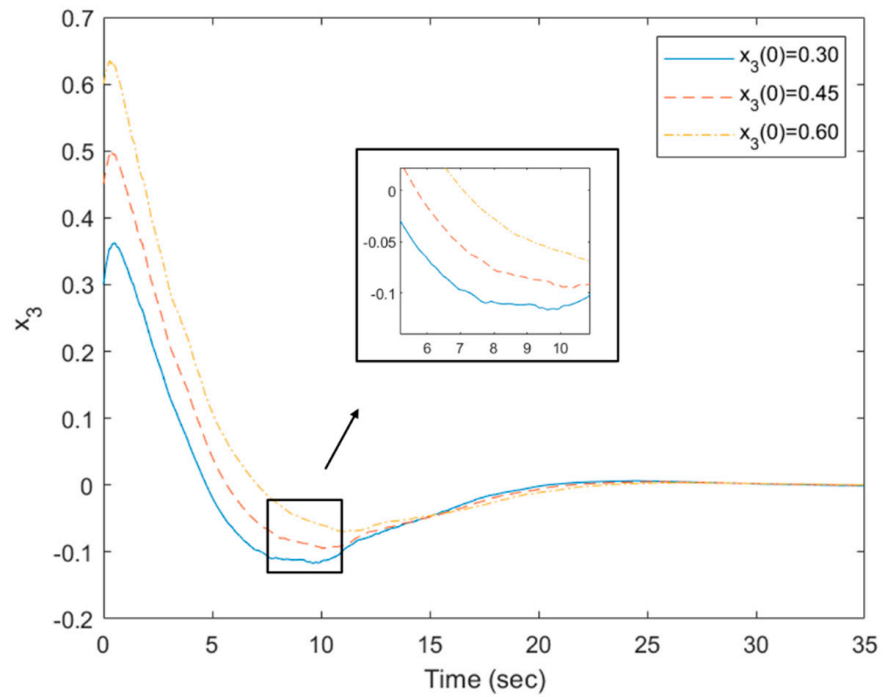

Figure 4. Response of $x_{3}(t)$. 


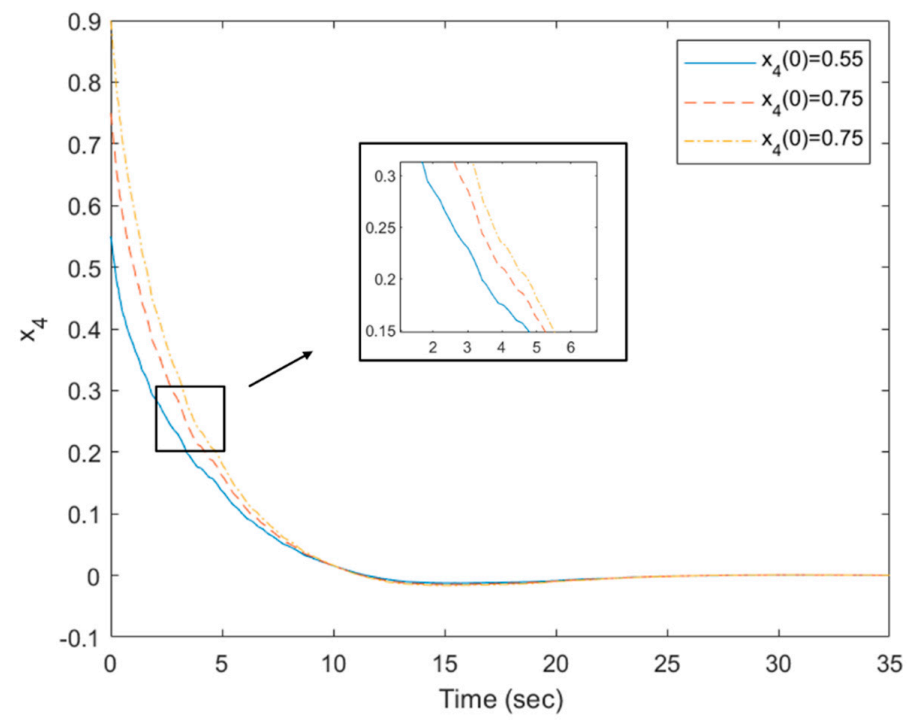

Figure 5. Response of $x_{4}(t)$.

In order to emphasize the advantage of the output feedback controller, the control method in [27] is applied to design a state-feedback controller. In [27], all states are assumed as measurable and applied to design the fuzzy controller. Based on the method of [27], the fuzzy controller can be designed as follows:

$$
u(t)=\sum_{i=1}^{2} h_{i}\left(x_{2}(t)\right)\left(-\mathbf{F}_{i} x(t)\right)
$$

where $\mathbf{F}_{1}=\left[\begin{array}{llll}-0.8670 & -0.0185 & -0.2540 & 0.0393\end{array}\right]$ and $\mathbf{F}_{2}=\left[\begin{array}{llll}-0.9364 & 0.3253 & 0.1446 & -0.0199\end{array}\right]$.

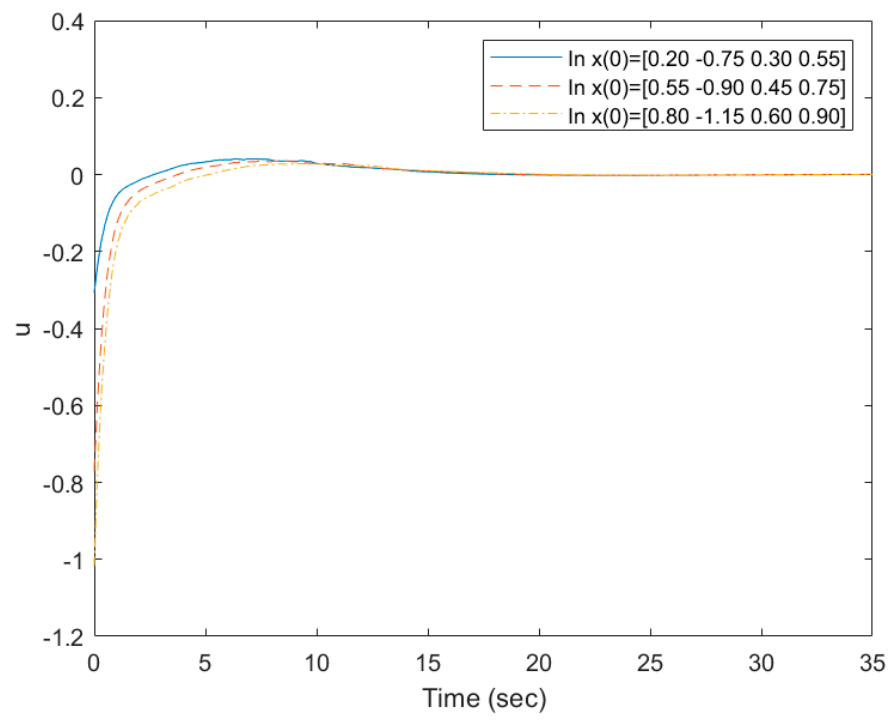

Figure 6. Response of $u(t)$.

With the fuzzy controller in (27) designed by [27], the response of $x_{1}(t)$ shown in Figure 7 cannot converge on zero. Therefore, the proposed design method is meaningful and practical for the control problem of nonlinear stochastic systems with unmeasurable states. Moreover, the asymptotical stability of nonlinear stochastic systems with unmeasurable states can be guaranteed by the proposed static output controller design method. 


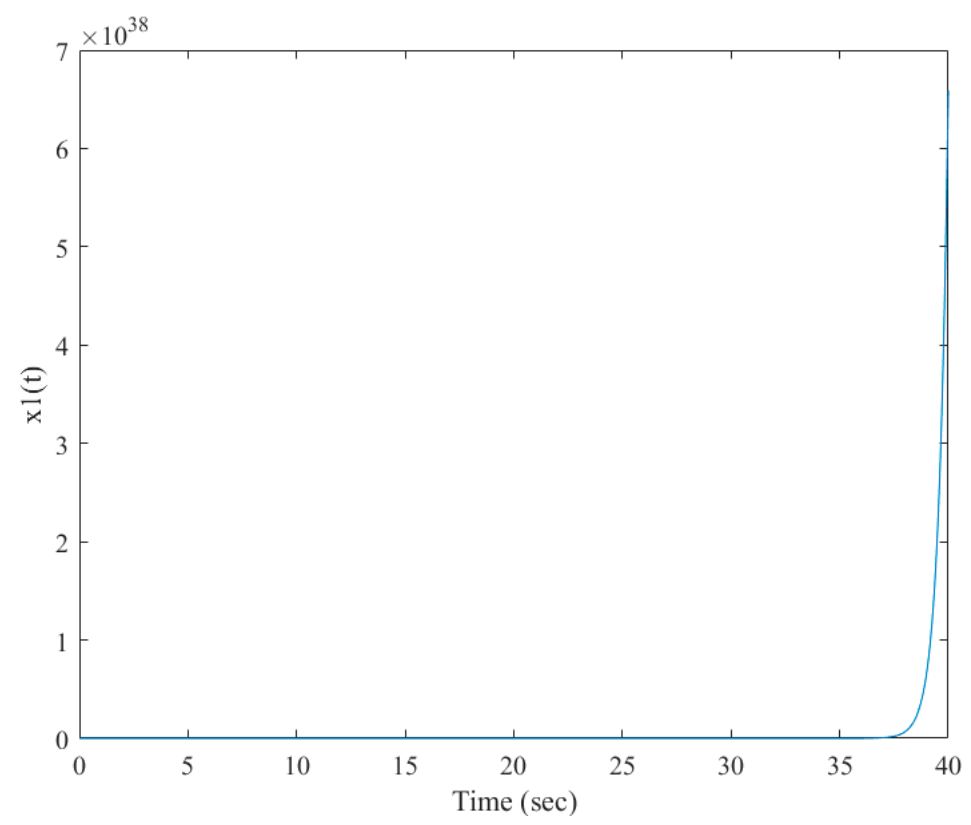

Figure 7. Response of $x_{1}(t)$ controlled by (27).

\section{Conclusions}

This paper addressed the static output control problem of nonlinear stochastic systems through application of the T-S fuzzy model and Itô's equation. Based on the PDC concept, the output controller was constructed with measured states to keep the relaxation of multiple matrices and, to avoid the derivative of the membership function, the LILF was applied to develop the stability criterion. Because of the structure of LILF, some lemmas were applied such that the sufficient condition could be solved via the convex optimization algorithm. Based on the proposed design method, some relaxations caused by LILF were demonstrated via the comparison in Case 1. When considering stochastic behavior, the proposed design method provides better transient performance than [18], as shown by Case 2 and Figure 1. Therefore, feasible solutions can be obtained to design an output controller to that guarantees the asymptotical stability of nonlinear stochastic systems in the mean square. We found that the output feedback controller is more feasible when dealing with a system with an unmeasurable state than the state-feedback controller, which is relevant to the discussion in [27].

Author Contributions: C.-C.K.: Conceptualization, C.-C.K. and Y.-C.Y.: Writing and editing, Y.-C.Y., Y.-H.L. and Y.-Y.H.: Software and data curation. All authors have read and agreed to the published version of the manuscript.

Funding: This research was funded by National Taiwan Ocean University grant number [NTOU109-006].

Institutional Review Board Statement: Not applicable.

Informed Consent Statement: Not applicable.

Data Availability Statement: All relevant data displayed in publication.

Conflicts of Interest: The authors declare no conflict of interest.

\section{References}

1. Chen, S.J.; Chen, S.M. Fuzzy Risk Analysis Based on Similarity Measures of Generalized Fuzzy Numbers. IEEE Trans. Fuzzy Syst. 2003, 11, 45-56. [CrossRef]

2. Zhumadillayeva, A.; Orazbayev, B.; Santeyeva, S.; Dyussekeyev, K.; Li, R.Y.M.; Crabbe, M.J.C.; Yue, X.G. Models for Oil Refinery Waste Management Using Determined and Fuzzy Conditions. Information 2020, 11, 299. [CrossRef] 
3. Takagi, T.; Sugeno, M. Fuzzy Identification of Systems and Its Applications to Modeling and Control. IEEE Trans. Syst. Man Cybern. 1985, 1, 116-132. [CrossRef]

4. Tanaka, K.; Sugeno, M. Stability Analysis and Design of Fuzzy Control Systems. Fuzzy Sets Syst. 1992, 45, 135-156. [CrossRef]

5. Huaping, L.; Kezhong, H.; Fuchun, S.; Zengpi, S. Analysis and Synthesis of Fuzzy Stochastic Systems via LMI Approach. In Proceedings of the IEEE Region 10 Conference on Computers, Communications, Control and Power Engineering, Beijing, China, 28-31 October 2002; Volume 3, pp. 1700-1703.

6. Wang, H.O.; Tanaka, K.; Griffin, M.F. An Approach to Fuzzy Control of Nonlinear Systems: Stability and Design Issue. IEEE Trans. Fuzzy Syst. 1996, 4, 14-23. [CrossRef]

7. Martínez-García, C.; Puig, V.; Astorga-Zaragoza, C.M.; Madrigal-Espinosa, G.; Reyes-Reyes, J. Estimation of Actuator and System Faults via an Unknown Input Interval Observer for Takagi-Sugeno Systems. Processes 2020, 8, 61. [CrossRef]

8. López-Estrada, F.R.; Rotondo, D.; Valencia-Palomo, G. A Review of Convex Approaches for Control, Observation and Safety of Linear Parameter Varying and Takagi-Sugeno Systems. Processes 2019, 7, 814. [CrossRef]

9. Nguyen, T.A.T.; Lauber, J.; Dambrine, M. Switching Fuzzy Control of the Air System of a Turbocharged Gasoline Engine. In Proceedings of the IEEE International Conference on Fuzzy Systems, Brisbane, Australia, 10-15 June 2012; pp. 1-7.

10. Ayoubi, M.A.; Swei, S.S.M.; Nguyen, N.T. Fuzzy Model-Based Pitch Stabilization and Wing Vibration Suppression of Flexible Aircraft. In Proceedings of the American Control Conference, Portland, OR, USA, 4-6 June 2014; pp. 3083-3088.

11. Ngongi, W.E.; Du, J.; Wang, R. Robust Fuzzy Controller Design for Dynamic Positioning System of Ships. Int. J. Control Autom. Syst. 2015, 13, 1294-1305. [CrossRef]

12. Wang, H.O.; Tanaka, K.; Griffin, M. Parallel Distributed Compensation of Nonlinear Systems by Takagi-Sugeno Fuzzy Model. In Proceedings of the IEEE International Conference on Fuzzy Systems, Yokohama, Japan, 20-24 March 1995; Volume 2, pp. 531-538.

13. Xie, W.B.; Li, H.; Wang, Z.H.; Zhang, J. Observer-Based Controller Design for a T-S Fuzzy System with Unknown Premise Variables. Int. J. Control Autom. Syst. 2019, 17, 907-915. [CrossRef]

14. Ku, C.C.; Chen, G.W. New Observer-Based Controller Design for LPV Stochastic Systems with Multiplicative Noise. Int. J. Robust Nonlinear Control 2019, 29, 4315-4327. [CrossRef]

15. Han, Z.X.; Feng, G.; Walcott, B.L.; Ma, J. Dynamic Output Feedback Controller Design for Fuzzy Systems. IEEE Trans. Syst. Man Cybern. 2000, 30, 204-210. [CrossRef]

16. Veselý, V. Static Output Feedback Controller Design. Kybernetika 2001, 37, 205-221.

17. Chadli, M.; Maquin, D.; Ragot, J. Static Output Feedback for Takagi-Sugeno Systems: An LMI Approach. In Proceedings of the 10th Mediterranean Conference on Control and Automation, Lisbon, Portugal, 9-12 July 2002.

18. Kau, S.W.; Lee, H.J.; Yang, C.M.; Lee, C.H.; Hong, L.; Fang, C.H. Robust $H_{\infty}$ Fuzzy Static Output Feedback Control of T-S Fuzzy Systems with Parametric Uncertainties. Fuzzy Sets Syst. 2007, 158, 135-146. [CrossRef]

19. Xie, W. Quadratic Stabilization of LPV System by an LTI Controller Based on ILMI Algorithm. Math. Probl. Eng. 2007, 2007, 208262. [CrossRef]

20. Huang, D.; Nguang, S.K. Static Output Feedback Controller Design for Fuzzy Systems: An ILMI Approach. Inf. Sci. 2007, 177, 3005-3015. [CrossRef]

21. Wu, H.N. An ILMI Approach to Robust $\mathrm{H}_{2}$ Static Output Feedback Fuzzy Control for Uncertain Discrete-Time Nonlinear Systems. Automatica 2008, 44, 2333-2339. [CrossRef]

22. Lam, H.K. Stability Analysis of T-S Fuzzy Control Systems Using Parameter-Dependent Lyapunov Function. IET Control Theory Appl. 2009, 3, 750-762. [CrossRef]

23. Xie, W. H2 Gain Scheduled State Feedback for LPV System with New LMI Formulation. IEE Proc. Control Theory Appl. 2005, 152, 693-697. [CrossRef]

24. Rhee, B.J.; Won, S. A New Fuzzy Lyapunov Function Approach for a Takagi-Sugeno Fuzzy Control System Design. Fuzzy Sets Syst. 2006, 157, 1211-1228. [CrossRef]

25. Zhao, X.; Lin, C.; Chen, B.; Wang, Q.G. A Novel Lyapunov-Krasovskii Functional Approach to Stability and Stabilization for T-S Fuzzy Systems with Time Delay. Neurocomputing 2018, 313, 288-294. [CrossRef]

26. Gonzalez, T.; Sala, A.; Bernal, M.; Robles, R. A Generalisation of Line-Integral Lyapunov Function for Takagi-Sugeno Systems. IFAC PapersOnLine 2017, 50, 2983-2988. [CrossRef]

27. Chang, W.J.; Chang, Y.C.; Ku, C.C. Passive Fuzzy Control via Fuzzy Integral Lyapunov Function for Nonlinear Ship Drum-Boiler Systems. J. Dyn. Syst. Meas. Control 2015, 137, 041008. [CrossRef]

28. Xing, S.; Zhu, B.; Zhang, Q. Stochastic Finite-Time Stabilization of a Class of Stochastic T-S Fuzzy System with the Itô's-Type. In Proceedings of the Chinese Control Conference, Xi'an, China, 26-28 July 2013; pp. 1570-1574.

29. Wu, L.; Ho, D.W. Fuzzy Filter Design for Itô Stochastic Systems with Application to Sensor Fault Detection. IEEE Trans. Fuzzy Syst. 2008, 17, 233-242.

30. Chang, W.J.; Chen, M.W.; Ku, C.C. Passive Fuzzy Controller Design for a Model Car via Discrete T-S Fuzzy Model with Multiplicative Noise. In Proceedings of the International Conference on Information and Automation, Zhuhai/Macau, China, 22-24 June 2009; pp. 1193-1198.

31. Xu, S.; Chen, T. $H_{\infty}$ Output Feedback Control for Uncertain Stochastic Systems with Time-Varying Delays. Automatica 2004, 40, 2091-2098. [CrossRef] 
32. Feron, E.; Apkarian, P.; Gahinet, P. Analysis and Synthesis of Robust Control Systems via Parameter-Dependent Lyapunov Functions. IEEE Trans. Autom. Control 1996, 41, 1041-1046. [CrossRef]

33. Boyd, S.; El Ghaoui, L.; Feron, E.; Balakrishnan, V. Linear Matrix Inequalities in System and Control Theory; Society for Industrial and Applied Mathematics: Philadelphia, PA, USA, 1994.

34. Lien, C.H.; Yu, K.W.; Chang, H.C. Mixed Performance Analysis of Continuous Switched Systems with Time-Varying Random Delay. Asian J. Control 2020, 22, 2156-2166. [CrossRef] 